\title{
Focus Group Results: Conclusion
}

In conclusion for patients, there is a lack of psychosexual care, which is very often compounded by male gender, ethnicity, and sometimes even healthcare professionals themselves. However, the focus group drew the conclusion psychosexual care can be well supported with a standardized opt out pathway of care starting after incontinence issues have been resolved. The healthcare professional interview results are analysed next, prior to drawing out the themes and developing the pathway. 